\title{
Safety of High-Flow Nasal Cannula Outside the ICU for Previously Healthy Children With Bronchiolitis
}

\author{
Nina M Dadlez, Nora Esteban-Cruciani, Asama Khan, Yi Shi, Kevin J McKenna, \\ Gabriella Azzarone, and William N Southern
}

\begin{abstract}
BACKGROUND: High-flow nasal cannula (HFNC), a form of noninvasive respiratory support, is effective for the treatment of respiratory distress in ICUs. Although HFNC has been used outside of the ICU, there is little research that examines its safety in this less-monitored setting. METHODS: Children $\leq \mathbf{2 4}$ months old admitted with bronchiolitis to a pediatric floor at a tertiary care center from April 1 2013, to March 31 2015, were identified by using standard diagnostic codes. Exclusion criteria were concomitant pneumonia or complex comorbidities. Demographic and clinical characteristics were abstracted. Outcomes included transfer to the ICU, higher levels of respiratory support, intubation, pneumothorax, or aspiration events. RESULTS: Eighty children admitted with bronchiolitis who were treated with HFNC while on the pediatric floor were examined. The median age was 4.6 months, $45 \%$ were girls, and the majority were either Hispanic $(41 \%)$ or black $(36 \%)$. Flow ranged from 3 to $10 \mathrm{~L} / \mathrm{min}$. Thirty-three subjects $(41 \%$ of the sample) required subsequent transfer to the ICU. No children were intubated or developed a pneumothorax. Eighty-three percent were fed while on HFNC. No children had an aspiration event. CONCLUSIONS: HFNC may be a safe modality of respiratory support outside of the ICU for children ages $\leq \mathbf{2 4}$ months with bronchiolitis and without comorbidities up to a maximum flow of $10 \mathrm{~L} / \mathrm{min}$. There were no adverse events among the subjects who were fed while on HFNC. Key words: high-flow nasal cannula; bronchiolitis; patient safety; noninvasive respiratory support; pediatric; complications. [Respir Care 2019;64(11):1410-1415. () 2019 Daedalus Enterprises]
\end{abstract}

\section{Introduction}

Viral bronchiolitis represents a significant burden of disease in the United States and accounts for $>150,000$ hospitalizations and more than $\$ 1.73$ billion in hospital charges annually. ${ }^{1,2}$ Although most children require only supportive care, $2-7 \%$ decompensate and require ventila-

Dr Dadlez is affiliated with the Division of Pediatric Hospital Medicine, Department of Pediatrics, Floating Hospital For Children at Tufts Medical Center, Tufts University School of Medicine, Boston, Massachusetts. Dr Esteban-Cruciani are affiliated with the Department of Pediatrics, St. Christopher's Hospital, Einstein Medical Center, Philadelphia, Pennsylvania. Dr Khan is affiliated with the Department of Surgery, State University of New York Upstate Medical University, Syracuse, New York. Dr Shi is affiliated with the Department of Pediatrics, Jacobi Medical Center, Albert Einstein College of Medicine, Bronx, New York. Dr McKenna is affiliated with the Department of Pediatrics, The Children's Hospital at Montefiore, Albert Einstein College of Medicine, Bronx, New York. Dr Azzarone is affiliated with Division of Pediatric Hospital Medicine, Department of Pediatrics, The Children's Hospital at Montefiore, Albert Einstein College of Medicine, Bronx, New York. Dr Southern tory support, which has traditionally required transfer to a pediatric ICU.2-4 High-flow nasal cannula (HFNC) is a therapeutic modality that has been effective in the treatment of respiratory failure in the ICU setting. ${ }^{5-11}$ Although HFNC has been used to treat bronchiolitis-associated respiratory failure outside of the ICU, there are concerns regarding the safety of using it in a less-monitored environment and the possibility that use of HFNC on the genis affiliated with the Department of Medicine, Montefiore Medical Cen-
ter, Albert Einstein College of Medicine Bronx, New York.

Supported by National Institutes of Health, National Center for Advancing Translational Science Einstein-Montefiore Clinical and Translational Science Award grant UL1TR001073.

The authors have disclosed no conflicts of interest.

Correspondence: Nina Dadlez MD MSc, Department of Pediatrics, Floating Hospital for Children at Tufts Medical Center, 800 Washington St\#7051, Boston, MA 02111. E-mail: NDadlez@tuftsmedicalcenter.org.

DOI: $10.4187 /$ respcare. 06352 


\section{HFNC FOR ChILDREN With BRONChIOLITIS}

eral unit might delay more-definitive airway management. To address this, we performed an observational cohort study of the safety of HFNC outside of the ICU setting.

Viral bronchiolitis is an acute infectious inflammatory condition of the airways, which causes significant respiratory distress in infants and young children. The care for bronchiolitis is primarily supportive, with suctioning and oxygen supplementation for mild-to-moderate bronchiolitis and/or noninvasive or invasive mechanical ventilation for children with severe bronchiolitis. ${ }^{12}$ HFNC provides heated and humidified oxygen and air at a variable flow. ${ }^{13}$ It delivers positive pressure at flows $>2 \mathrm{~L} / \mathrm{min}$ and is thought to improve alveolar ventilation by $\mathrm{CO}_{2}$ washout of airway dead space. ${ }^{13}$ Several randomized controlled trials of the use of HFNC in the neonatal ICU demonstrated noninferiority and similar safety profiles when compared with CPAP as a means of primary respiratory support for premature infants and as transitional support after extubation. ${ }^{5-8,14}$ Recent studies in the pediatric ICU and pediatric emergency department patient populations found that the use of HFNC in children with respiratory distress is associated with improved oxygen saturation and respiratory scale scores, and a decreased need for intubation. ${ }^{9-11}$

Studies to date have focused on the use of HFNC in intensive care and emergency department settings, whereas few studies examined the use of HFNC in general pediatric inpatient units. ${ }^{15}$ Despite a lack of evidence that demonstrates safety and effectiveness, HFNC is now being used to treat children with bronchiolitis outside of the ICU setting. ${ }^{15,16}$ General pediatric units are a less-monitored environment than the ICU, with lower nursing-patient ratios and less support from respiratory therapists. The safety of HFNC in this lessmonitored and lower resource setting has not been established. There have also been case reports of air-leak syndromes (eg, pneumothorax, pneumomediastinum, or subcutaneous emphysema) with the use of HFNC. ${ }^{17}$ Furthermore, although some children on HFNC have been fed in a highly monitored setting, it is unclear whether enteral nutrition is safe while children are on HFNC or whether parenteral hydration and nutrition is necessary to reduce the risk of aspiration. ${ }^{18,19}$ We sought to evaluate the feasibility and safety of HFNC for the treatment of bronchiolitis outside the ICU. In addition, we examined the feeding practices and safety of feeding children treated with HFNC. We hypothesized that the use of HFNC outside of the ICU would not be associated with pneumothorax, aspiration, intubation, or death.

\section{Methods}

\section{Overview}

This was a retrospective cohort study that examined the safety of the use of HFNC outside of the ICU in children with bronchiolitis. The Children's Hospital at Montefiore

\section{QUICK LOOK}

\section{Current knowledge}

High-flow nasal cannula (HFNC) is an effective form of noninvasive respiratory support in the intensive care setting, and studies reported noninferiority and similar safety profiles when compared with CPAP. A Cochrane review in 2014 called for additional evidence of the safety of HFNC for infants with bronchiolitis, and data are lacking on the use of this modality outside of the ICU setting.

\section{What this paper contributes to our knowledge}

This study indicated that HFNC may be a safe modality of respiratory support for previously healthy children with bronchiolitis outside of the ICU. However, pediatric ICU transfer was common and, because the majority of children transferred in the first $24 \mathrm{~h}$ after initiating HFNC, close observation was warranted during this time. In addition, there were no adverse events among the subjects who were fed orally while on HFNC.

is a 132-bed academic tertiary care children's hospital that is part of a 4-hospital health center. The hospital is located in the Bronx, New York, and cares for an underserved patient population. This study was approved by the institutional review board at Montefiore Medical Center.

\section{Implementing HFNC on a General Pediatrics Unit}

In April 2013, our institution initiated the use of HFNC outside of the ICU for children with bronchiolitis without comorbidities. Before this, HFNC was only administered in the pediatric ICU. A pediatric unit on which the majority of infants and toddlers are cared for was chosen as the single site for implementation of HFNC due to the prevalence of bronchiolitis in this population. An HFNC protocol was developed and disseminated that specified the clinical criteria for HFNC and outlined the roles of all team members who cared for the patient. One-hour didactic training was required for hospital medicine attending physicians, resident physicians, and nurses who worked on the identified unit, led by pediatric ICU faculty, respiratory therapists, and nurse educators. The training sessions were designed to teach the mechanics and physiology of HFNC and the ways that HFNC can be used in the support of patients with severe viral bronchiolitis. At the time of HFNC implementation, a dedicated respiratory therapist was assigned to the unit. Respiratory therapists were responsible for setting up the HFNC and conducting rounds on patients on HFNC to monitor their clinical status. Nurs- 
ing ratios for the nurses caring for a patient on HFNC were adjusted from a 1:5 to a 1:3 nursing ratio and a nurse could only care for one patient on HFNC at a time. A decision to start a patient on HFNC was at the discretion of the primary provider and was based on an overall clinical picture, including factors such as the patient's breathing frequency and accessory muscle use. Clinicians who made this decision were unaware of a study of HFNC safety.

\section{HFNC Delivery}

The HFNC support mechanism consisted of a low-flow meter, blender, and heating humidification system. The use of HFNC was documented in the charts by respiratory therapists and HFNC was initiated at 4 or $6 \mathrm{~L} / \mathrm{min}$ based on the age of the patient, and adjusted based on the clinical response. For the purposes of our study, HFNC was defined as the use of the above system as documented by respiratory therapy. Use of traditional wall nasal cannula at any rate without the HFNC hardware was not considered to be HFNC.

\section{Study Participants}

Children $\leq 24$ months old who were admitted to the general pediatric infant and toddler unit at an urban tertiary care center with a diagnosis of bronchiolitis between April 1, 2013, and March 31, 2015, were included in the study. Patients were identified by a primary ICD-9 discharge diagnosis of acute bronchiolitis, acute bronchiolitis due to respiratory syncytial virus, and acute bronchiolitis due to other infectious organism or a primary diagnosis of acute respiratory failure with a secondary diagnosis of bronchiolitis (codes 466.11 and 466.19). Children who received HFNC on the pediatric unit were included in the study and those who did not receive HFNC or who went directly to the pediatric ICU were excluded. Because children with complex comorbidities that impact respiratory status are not eligible for HFNC outside of the ICU at our institution, our sample did not include children diagnosed with a concomitant bacterial pneumonia or with cardiac, pulmonary, neurologic, metabolic, or craniofacial comorbidities.

\section{Data Collection}

Demographic and admission characteristics for each subject were extracted from Montefiore's Clinical Information System by using Clinical Looking Glass (Streamline Health, Atlanta, Georgia), a data extraction user interface used for research and quality improvement. Clinical history, examination findings, respiratory support, and outcomes were obtained via a concomitant review of electronic charts. Ten percent of all records were examined by a second reviewer (ND) to ensure accuracy.

\section{HFNC Measures}

HFNC utilization measures were collected for each subject. These included maximum and minimum flow, maximum $\mathrm{F}_{\mathrm{IO}_{2}}$ used, and the duration of time on HFNC. HFNC measures were collected while the subject was on the general pediatric unit and did not include HFNC administered in the pediatric ICU.

\section{Safety Measures}

Safety measures included the need for transfer to the pediatric ICU, escalation to higher levels of respiratory support (CPAP, noninvasive ventilation, bilevel positive airway pressure, or intubation), clinically important pneumothorax, aspiration, and death. Reasons for transfer to the pediatric ICU included escalation of respiratory support and the need for closer clinical monitoring. A decision to transfer was at the discretion of the primary provider in consultation with the ICU. Clinicians who made the decisions to transfer were unaware of a study of the safety of HFNC. All available imaging reports for each child were reviewed to assess for the presence of a pneumothorax. Children who did not have imaging after initiation of HFNC were presumed not to have a clinically important pneumothorax. Each subject's hospital course was reviewed to determine if there was an aspiration event during admission. An aspiration event was defined as aspiration mentioned in the clinical notes or a new infiltrate noted on a chest radiograph.

\section{Feeding Practices}

The charts were reviewed to determine if a subject was fed orally, via nasogastric tube, or not fed while on HFNC. Feeding while on HFNC was considered a dichotomous variable in which children fed at any time while on HFNC were considered to have been fed. The decision to feed and the modality of feeding (orally or via nasogastric tube) was at the discretion of the primary provider and based on the overall clinical picture, including factors such as the patient's breathing frequency, accessory muscle use, and oxygenation.

\section{Statistical Analysis}

Descriptive statistics and frequency histograms were used to examine the demographic and clinical characteristics of the study population. To compare demographic and clinical characteristics of children who required HFNC versus children who did not require HFNC and children who were fed orally versus children not fed orally, chisquare and Wilcoxon rank-sum tests were performed. All 


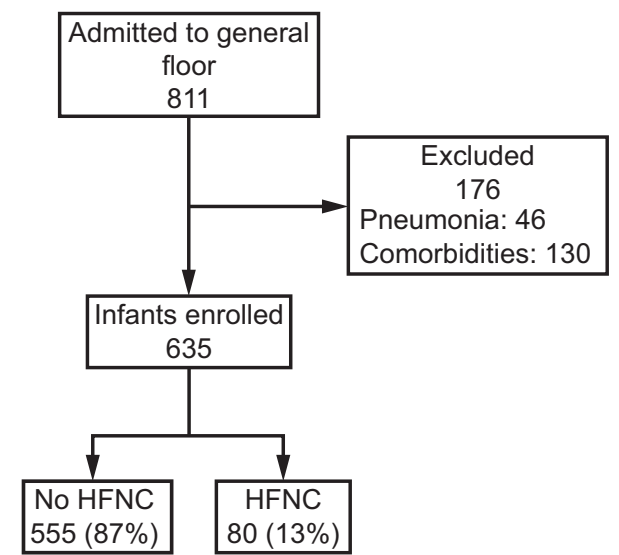

Fig. 1. Flow chart. HFNC = high-flow nasal cannula.

Table 1. Participant Characteristics

\begin{tabular}{lc}
\hline \multicolumn{1}{c}{ Characteristic } & Value \\
\hline Girls, $n(\%)$ & $36(45)$ \\
Age, median (interquartile range) mo & $4.6(2.0-10.4)$ \\
Race, $n(\%)$ & \\
$\quad$ Hispanic & $33(41.2)$ \\
Black & $29(36.2)$ \\
White & $3(3.8)$ \\
Other/unknown & $15(18.8)$ \\
Medicaid, $n(\%)$ & $67(83.8)$ \\
Premature, $n(\%)$ & $19(23.5)$ \\
Previous respiratory admission, $n(\%)$ & $12(15.0)$ \\
Previous respiratory admission to the & $4(5.1)$ \\
$\quad$ pediatric ICU, $n(\%)$ & \\
\hline$N=80$. & \\
\hline
\end{tabular}

analyses were performed by using Stata 13.0 (StataCorp, College Station, Texas).

\section{Results}

\section{Participants}

A total of 945 children were admitted to the pediatric in-patient unit for bronchiolitis. Of these, 310 were excluded (Fig. 1). Of the remaining 635, 80 children (13\%) were treated with HFNC. Of the study population, $45 \%$ were girls and the majority had Medicaid as their primary insurance (84\%). The study population was $41 \%$ Hispanic, $36 \%$ black/African-American, and 3.8\% white (Table 1). The median (interquartile range [IQR]) age was 4.6 (2.0$10.4)$ months. Twenty-four percent had a history of prematurity (24\%). The median (IQR) in-patient stay was $6.1(5.2-7.9) \mathrm{d}$ from the time of emergency department triage to discharge.
Table 2. Safety Metrics for the Use of HFNC Outside of the ICU

\begin{tabular}{lc}
\hline \hline \multicolumn{1}{c}{ Metric } & Results, $n(\%)$ \\
\hline Pediatric ICU transfer & $33(41)$ \\
Higher noninvasive respiratory support & $19(24)$ \\
Intubation & $0(0)$ \\
Pneumothorax & $0(0)$ \\
Aspiration & $0(0)$ \\
Death & $0(0)$ \\
& \\
$N=80$. & \\
HFNC $=$ high-flow nasal cannula & \\
\hline
\end{tabular}

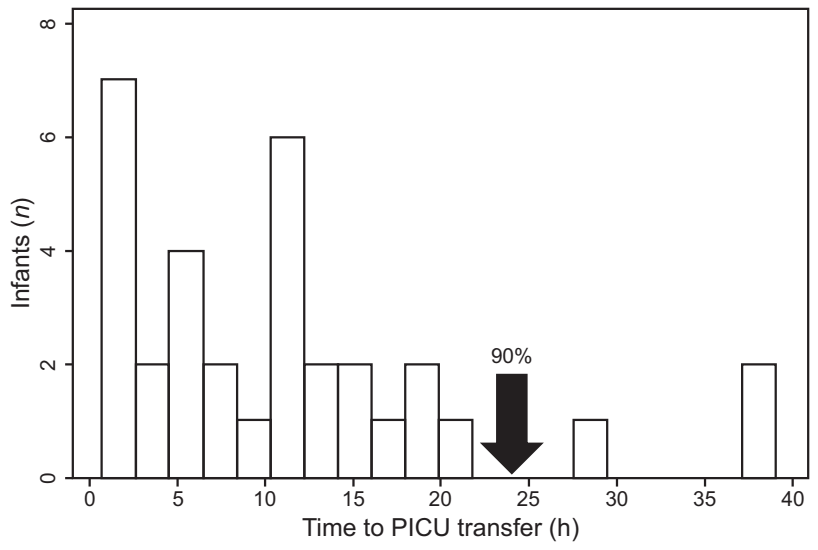

Fig. 2. Time to pediatric ICU (PICU) transfer after initiation of highflow nasal cannula.

\section{HFNC Measures}

The median (IQR) minimum flow was $3(3-5) \mathrm{L} / \mathrm{min}$. The maximum flow was $10 \mathrm{~L} / \mathrm{min}$. The median (IQR) maximum flow for all children was $8(6-8) \mathrm{L} / \mathrm{min}$. Among children who stayed on the general floor, the median (IQR) maximum flow was 7 (6-8) $\mathrm{L} / \mathrm{min}$, and, among children who were transferred to the pediatric ICU, the median (IQR) maximum flow was $8(8-10) \mathrm{L} / \mathrm{min}$, which represented a significant difference $(P<.001)$. The median (IQR) maximum $\mathrm{F}_{\mathrm{IO}_{2}}$ was $0.40(0.30-0.50)$.

\section{Safety Measures}

Of the children treated with HFNC outside of the ICU, $41 \%$ required transfer to the pediatric ICU and 59\% stayed on the general pediatric unit (Table 2). Among the children who stayed on the pediatric floor, the median (IQR) time spent on HFNC was $3.0(2.0-4.2)$ d. Among the children who required transfer to the pediatric ICU, 30 (91\% of those who required pediatric ICU transfer) were transferred in the first $24 \mathrm{~h}$ after initiation of HFNC (Fig. 2) and $58 \%$ required escalation to higher levels of respiratory support after transfer. There were no intubations. No chil- 
Table 3. Feeding While on HFNC

\begin{tabular}{lc}
\hline \hline \multicolumn{1}{c}{ Parameter } & Results, $n(\%)^{*}$ \\
\hline Fed while on HFNC & $66(82.5)$ \\
Regular oral & $52(78.8)$ \\
Clears only & $13(19.7)$ \\
$\quad$ Nasogastric tube only & $1(1.5)$ \\
Not fed while on HFNC & $14(17.5)$ \\
& \\
\hline$N=80$. & \\
$*$ Due to rounding, percentages may not add up to $100 \%$. & \\
HFNC = high-flow nasal cannula & \\
\end{tabular}

dren had a clinically important pneumothorax, and there were no aspiration events. There were no deaths.

\section{Feeding Practices}

The majority of children $(82.5 \%)$ in the cohort were fed while on HFNC. Of the children who were fed, the primary modality was oral feeds $(98.5 \%)$ and only 1 child was fed via a nasogastric tube only $(1.5 \%)$. Of the children who were fed, $78.8 \%$ received their full oral diet (breast milk, formula, and/or purees) and $19.7 \%$ received clear diet only (Table 3). No subjects had an aspiration event. Of the 14 subjects who were not fed, all (100\%) required transfer to the pediatric ICU.

\section{Discussion}

In this observational cohort study, we examined 80 children with acute bronchiolitis who were treated with HFNC outside of the ICU. Of these, $41 \%$ required transfer to the pediatric ICU, most within $24 \mathrm{~h}$ of initiation of HFNC. No children who received HFNC on the general pediatric unit developed pneumothorax, required intubation, or died. A majority $(82.5 \%)$ were fed, and there were no aspiration events. Overall, results of our study indicated that the use of HFNC was safe when used outside of the ICU for healthy children with bronchiolitis with the required infrastructure in place.

Studies that examined the safety of HFNC to date primarily focused on its safety compared with other modalities of noninvasive respiratory support, particularly CPAP. ${ }^{5,6,8,20,21}$ Several randomized controlled trials of HFNC versus CPAP in premature infants as a means of primary respiratory support or transitional support after extubation showed no difference in rates of pneumothorax or deaths. ${ }^{5,6,8,20,21}$ However, these studies do not imply that HFNC is safe to use outside of the ICU, where event rates would be expected to be lower and CPAP is generally considered an unacceptable risk. In one observational study of use of HFNC in infants with bronchiolitis in the pediatric ICU setting, 2 infants developed pneumothorax. ${ }^{11}$
Although this may be an acceptable event rate in the ICU, it calls into question whether HFNC is safe enough for healthy children outside of the pediatric ICU.

For this reason, a Cochrane review in 2014 on the use of the HFNC for children with bronchiolitis concluded that further evidence of the safety of HFNC in this population is required. ${ }^{22}$ Since then, Riese et al ${ }^{15}$ examined the effect of implementation of an HFNC protocol on length of stay and their work indicated that HFNC is safe; however, they were unable to assess the safety outcomes of individual children started on HFNC when on the floor. A second study examined the safety of HFNC in a community hospital setting; however, the primary safety outcome was transfer to a tertiary care facility. ${ }^{16}$ The present study was the largest cohort that focused solely on children treated with HFNC outside of the ICU and, to our knowledge, the first study to examine the safety of feeding during HFNC treatment in this setting.

Although $41 \%$ of children in our cohort required transfer to the pediatric ICU, they did not develop adverse outcomes (pneumothorax, intubation, or death), which indicated that initial management outside of the ICU did not cause harm. This high transfer rate suggested that the availability of pediatric intensive care services should be a factor for institutions when considering initiation of HFNC outside of the ICU. We found that $90 \%$ of children who required transfer to the pediatric ICU were transferred within $24 \mathrm{~h}$ of initiation of HFNC, which indicated that this was likely a critical time period for monitoring. However, it should be noted that, in our cohort, $59 \%$ of children initiated on HFNC on the pediatric unit were successfully managed without transfer to an ICU. Before the availability of HFNC on the pediatric unit, all the subjects on HFNC would have been managed in the pediatric ICU. Therefore, use of HFNC outside of the ICU setting may enable some children to safely avoid a pediatric ICU admission.

There are very few studies that examined the safety of enteral feeding while children are being treated with HFNC. Positive pressure provided by HFNC may disrupt children's suck-swallow-breathe reflex, predisposing them to aspiration. ${ }^{23}$ One study of premature infants on HFNC for respiratory distress syndrome in the neonatal ICU found that the 17 infants in their cohort who were fed orally did not show overt signs of cough or dysphagia. ${ }^{24}$ However, this was a small sample size and findings from this patient population may be different from children ages $0-2$ y and with bronchiolitis. Two studies that examined feeding in patients with bronchiolitis on HFNC in the pediatric ICU found 1 patient with aspiration-related respiratory failure ( $0.8 \%$ of the cohort) and a low incidence of feeding-related adverse events. ${ }^{18,19}$ In our study, the majority of children (82.5\%) were fed orally while on HFNC and there were no aspiration events. This indicated that feeding orally on HFNC was safe. Of note, the children who 


\section{HFNC FOR ChILDREN With BRONChIOLITIS}

were not fed orally were all subsequently transferred to the pediatric ICU, which indicated that providers were adept at assessing which children are at risk for requiring higher levels of respiratory support, which would change their aspiration risk.

Our study had several limitations. It was a retrospective chart review at a single institution. In addition, our setting was an urban tertiary care center with an on-site pediatric ICU and respiratory therapists available on the unit, so our experience may not be generalizable to institutions that do not have these resources available. In addition, our results were focused on otherwise healthy children with bronchiolitis and may not be applicable to children with comorbidities. Our cohort included a large proportion of Hispanic patients, which may not be reflective of the population at other institutions. Our assessment of aspiration events may be limited because some patients may have been determined by their providers to have been too high risk to feed. Finally, our study assessed only clinically important aspiration events and did not capture microaspiration events that may not be detected by providers.

\section{Conclusions}

HFNC may be a safe modality of respiratory support for children with bronchiolitis without complex comorbidities outside of the ICU setting with the required resources available, to a maximum flow of $10 \mathrm{~L} / \mathrm{min}$. Pediatric ICU transfer was common, and the majority of children were transferred in the first $24 \mathrm{~h}$ after HFNC was initiated, which suggested that close observation is warranted during this time. In addition, there were no adverse events among the subjects who were fed orally while on HFNC. Further research to assess the effectiveness of HFNC outside of the ICU to prevent transfer to the pediatric ICU and the need for higher levels of respiratory support is needed.

\section{REFERENCES}

1. HCUP Kids' Inpatient Database (KID). Healthcare Cost and Utilization Project (HCUP). Rockville, MD; Agency for Healthcare Research and Quality; 2012. Available at http://www.hcup-us.ahrq.gov/ kidoverview.jsp. Accessed January 21, 2015.

2. Hasegawa K, Tsugawa Y, Brown DF, Mansbach JM, Camargo CA Jr. Trends in bronchiolitis hospitalizations in the United States, 20002009. Pediatrics 2013;132(1):28-36.

3. Chan PW, Lok FY, Khatijah SB. Risk factors for hypoxemia and respiratory failure in respiratory syncytial virus bronchiolitis. Southeast Asian J Trop Med Public Health 2002;33(4):806-810.

4. Outwater KM, Crone RK. Management of respiratory failure in infants with acute viral bronchiolitis. Am J Dis Child 1984;138(11): 1071-1075.

5. Collins CL, Holberton JR, Barfield C, Davis PG. A randomized controlled trial to compare heated humidified high-flow nasal can- nulae with nasal continuous positive airway pressure postextubation in premature infants. J Pediatr 2013;162(5):949-954.e1.

6. Manley BJ, Owen LS, Doyle LW, Andersen CC, Cartwright DW, Pritchard MA, et al. High-flow nasal cannulae in very preterm infants after extubation. N Engl J Med 2013;369(15):1425-1433.

7. Saslow JG, Aghai ZH, Nakhla TA, Hart JJ, Lawrysh R, Stahl GE, Pyon KH. Work of breathing using high-flow nasal cannula in preterm infants. J Perinatol 2006;26(8):476-480.

8. Yoder BA, Stoddard RA, Li M, King J, Dirnberger DR, Abbasi S. Heated, humidified high-flow nasal cannula versus nasal CPAP for respiratory support in neonates. Pediatrics 2013;131(5):e1482-1490.

9. Spentzas T, Minarik M, Patters AB, Vinson B, Stidham G. Children with respiratory distress treated with high-flow nasal cannula. J Intensive Care Med 2009;24(5):323-328.

10. Wing R, James C, Maranda LS, Armsby CC. Use of high-flow nasal cannula support in the emergency department reduces the need for intubation in pediatric acute respiratory insufficiency. Pediatr Emerg Care 2012;28(11):1117-1123.

11. McKiernan C, Chua LC, Visintainer PF, Allen H. High flow nasal cannulae therapy in infants with bronchiolitis. J Pediatr 2010;156(4): 634-638.

12. American Academy of Pediatrics Subcommittee on Diagnosis and Management of Bronchiolitis. Diagnosis and management of bronchiolitis. Pediatrics 2006;118(4):1774-1793

13. Haq I, Gopalakaje S, Fenton AC, McKean MC, J O'Brien C, Brodlie M. The evidence for high flow nasal cannula devices in infants. Paediatr Respir Rev 2014;15(2):124-134.

14. Manley BJ, Dold SK, Davis PG, Roehr CC. High-flow nasal cannulae for respiratory support of preterm infants: a review of the evidence. Neonatology 2012;102(4):300-308.

15. Riese J, Porter T, Fierce J, Riese A, Richardson T, Alverson BK. Clinical outcomes of bronchiolitis after implementation of a general ward high flow nasal cannula guideline. Hosp Pediatr 2017;7(4):197-203.

16. Davison M, Watson M, Wockner L, Kinnear F. Paediatric high-flow nasal cannula therapy in children with bronchiolitis: a retrospective safety and efficacy study in a non-tertiary environment. Emerg Med Australas 2017;29(2):198-203.

17. Hegde S, Prodhan P. Serious air leak syndrome complicating highflow nasal cannula therapy: a report of 3 cases. Pediatrics 2013; 131(3):e939-944.

18. Sochet AA, McGee JA, October TW. Oral nutrition in children with bronchiolitis on high-flow nasal cannula is well tolerated. Hosp Pediatr 2017;7(5):249-255.

19. Slain KN, Martinez-Schlurmann N, Shein SL, Stormorken A. Nutrition and high-flow nasal cannula respiratory support in children with bronchiolitis. Hosp Pediatr 2017;7(5):256-262.

20. Kotecha SJ, Adappa R, Gupta N, Watkins WJ, Kotecha S, Chakraborty M. Safety and efficacy of high-flow nasal cannula therapy in preterm infants: a meta-analysis. Pediatrics 2015;136(3):542-553.

21. Holleman-Duray D, Kaupie D, Weiss MG. Heated humidified highflow nasal cannula: use and a neonatal early extubation protocol. J Perinatol 2007;27(12):776-781.

22. Beggs S, Wong ZH, Kaul S, Ogden KJ, Walters JA. High-flow nasal cannula therapy for infants with bronchiolitis. Cochrane Database Syst Rev 2014;(1):CD009609.

23. Dodrill P, Gosa M, Thoyre S, Shaker C, Pados B, Park J, et al. First, do no harm: a response to "oral alimentation in neonatal and adult populations requiring high-flow oxygen via nasal cannula". Dysphagia 2016;(6):781-782.

24. Leder SB, Siner JM, Bizzarro MJ, McGinley BM, Lefton-Greif MA. Oral alimentation in neonatal and adult populations requiring high-flow oxygen via nasal cannula. Dysphagia 2016;31(2): 154-159. 